\title{
Absorption systems in radio-selected QSO surveys
}

\author{
Sara L. Ellison ${ }^{1}$ \\ ${ }^{1}$ University of Victoria, Dept. Physics \& Astronomy, Victoria, BC, V8P 1A1, Canada \\ email: sarae@uvic.ca
}

\begin{abstract}
Radio-selected samples of quasars with complete optical identifications offer an ideal dataset with which to investigate dust bias associated with intervening absorption systems. Here, we review our work on the Complete Optical and Radio Absorption Line System (CORALS) survey whose aim is to quantify this bias and assess the impact of dust on absorber statistics. First, we review previously published results on the number density and gas content of high column density absorbers over the redshift range $0.6<z<3.5$. We then present the latest results from CORALS which focus on measuring the metal content of our unbiased absorber sample and an investigation of their optical-IR colours. Overall we find that although dust is unarguably present in absorption galaxies, the level appears to be low enough that the statistics of previous magnitude limited samples have not been severely affected and that the subsequent reddening of background QSOs is small.
\end{abstract}

\section{Introduction}

The issue of dust bias in QSO absorption line surveys is as old as the study of damped Lyman alpha systems (DLAs) themselves, dating back some two decades to the mid 1980s (e.g. Ostriker \& Heisler 1984). However, it is evident from the number of contributions at this conference that the dust content of DLAs and its impact on our observations remains a debated and relevant issue. The question can be simply stated: is there a sizable population of DLAs with enough dust to dim background QSOs and cause them to drop out of magnitude limited samples? This question is pivotal for the interpretation of DLA statistics since QSO absorption lines are usually heralded as a 'representative probe' of the gas in the high redshift Universe. This is true, in the sense that we do not rely on starlight to detect DLA galaxies. However, if our observations are biased against chemically evolved systems with high dust fractions, the lore that underpins the utility of DLAs as early galaxy probes will have to be revised. Under various assumptions for the chemical composition and dust properties of high redshift galaxies, a number of theoretical models have advocated dust-induced selection effects (e.g. Ostriker \& Heisler 1984; Fall \& Pei 1993; Churches et al. 2004; Vladilo \& Péroux 2005). The predicted impact on observations is many-fold and ranges from under-estimates of the absorber number density and total neutral gas density, to the metallicities inferred from volatile elements such as Zn. In addition to this theoretical impetus, it is tempting to appeal to dust bias in order to explain a number of observational trends, such as the anti-correlation between $\mathrm{N}(\mathrm{HI})$ and $[\mathrm{Zn} / \mathrm{H}]$ (Boissé et al. 1998) and the very mild evolution of the mean metallicity over $0.5<z<3.5$ (e.g. Kulkarni et al. 2005).

In order to quantify the effect of dust induced biases in previous surveys for DLAs, it is necessary to construct a sample of QSOs that is optically complete and has no magnitude limit. One way to achieve this is to select QSOs at wavelengths that are not affected by the presence of dust, such as in the radio or X-ray regimes, and then conduct deep optical observations to identify their counterparts. Any survey for intervening 
absorbers based on such a sample will be free from extinction induced biases and its statistics can be compared with magnitude limited samples. This simple strategy has formed the crux of the Complete Optical and Radio Absorption Line System (CORALS) survey for absorbers in the radio-selected Parkes 0.25 Jy (PQJ) QSO sample of Jackson et al. (2002). Follow-up optical observations (combined with position matching in existing digitised surveys) resulted in optical counterparts for every QSO in the PQJ sample, making it an ideal sample with which to investigate dust bias.

\section{Results from CORALS}

2.1. High redshift DLAs - number density and neutral gas content at $1.8<z<3.5$

The first results from CORALS were published by Ellison et al. (2001); we only briefly review the main results of that work here and reserve the majority of this proceedings for newer results and a discussion of the survey's implications. In short, Ellison et al. (2001) conducted a search for DLAs with $z_{\text {abs }}>1.8$ toward the $66 z_{\mathrm{em}}>2.2$ QSOs in the PQJ sample, covering a total redshift path of $\Delta z \sim 55$. The number density of DLAs per unit redshift $(n(z))$ and the neutral gas mass density (expressed as a fraction of the closure density, $\Omega_{\mathrm{DLA}}$ ) were both found to be consistent with previous surveys, indicating that dust is not a significant bias in optically selected samples. However, Ellison et al. (2001) also tentatively pointed out a mild dependence of $n(z)$ and $\Omega_{\mathrm{DLA}}$ on QSO magnitude, although this dependence seemed to flatten at magnitudes fainter than $B \sim 19.5$ (see also the poster by Smette et al. at this meeting). In Ellison et al. (2004) we speculated that this may be due to a combination of a small amount of extinction (i.e. not enough to affect the statistics of the sample as a whole) and the shape of the QSO optical luminosity function.

\subsection{Low redshift MgII systems - inferences on DLA number density at $0.6<z<1.7$}

Although the majority of known DLAs are at $z>1.7$, this is not where we might expect dust bias to be most severe. It is true that galaxies with large dust masses have been detected at $z>2$ with sub-mm instruments such as SCUBA. However, it seems unlikely that low mass stars, the usual culprits for dust formation at low $z$, can have significantly contributed to high redshift galaxies. By $z<1.5$, on the other hand, active star formation has been on-going at high rates for some 4 billion years, so we may expect that galaxies have accrued significantly more dust by these epochs.

Conducting a low redshift survey analogous to the high redshift campaign described in the previous section is impractical due to HST's small aperture (and now its complete lack of a UV spectrograph). However, much can be learned from a ground based survey of strong metal line systems. As Sandhya Rao has described at this meeting, targeting absorbers with high rest equivalent widths (EW > $0.5 \AA$ ) of MgII $\lambda 2796$ and FeII $\lambda 2600$ is an efficient way of pre-selecting DLA candidates (see also Rao \& Turnshek 2000). We have therefore searched for strong MgII systems toward our sample of radio-selected QSOs over the redshift range $0.6<z_{\text {abs }}<1.7$. Our sample is slightly different from the high $z$ sample described in the previous section, in that we observed QSOs in the range $1.80<z_{\mathrm{em}}<2.55$. This modification has been made so that we optimise our redshift coverage of MgII systems which will lie red-wards of the QSO's Ly $\alpha$ emission. Out of 75 QSOs we have identified $47 \mathrm{MgII}$ absorbers with EW $>0.3 \AA$. We compare the number density of MgII systems in our low redshift CORALS survey to the SDSS sample of Nestor, Turnshek \& Rao (2005) in Fig. 1. The agreement is excellent, indicating that our complete survey has not uncovered a large population of MgII absorbers that have been previously overlooked by magnitude limited samples. 

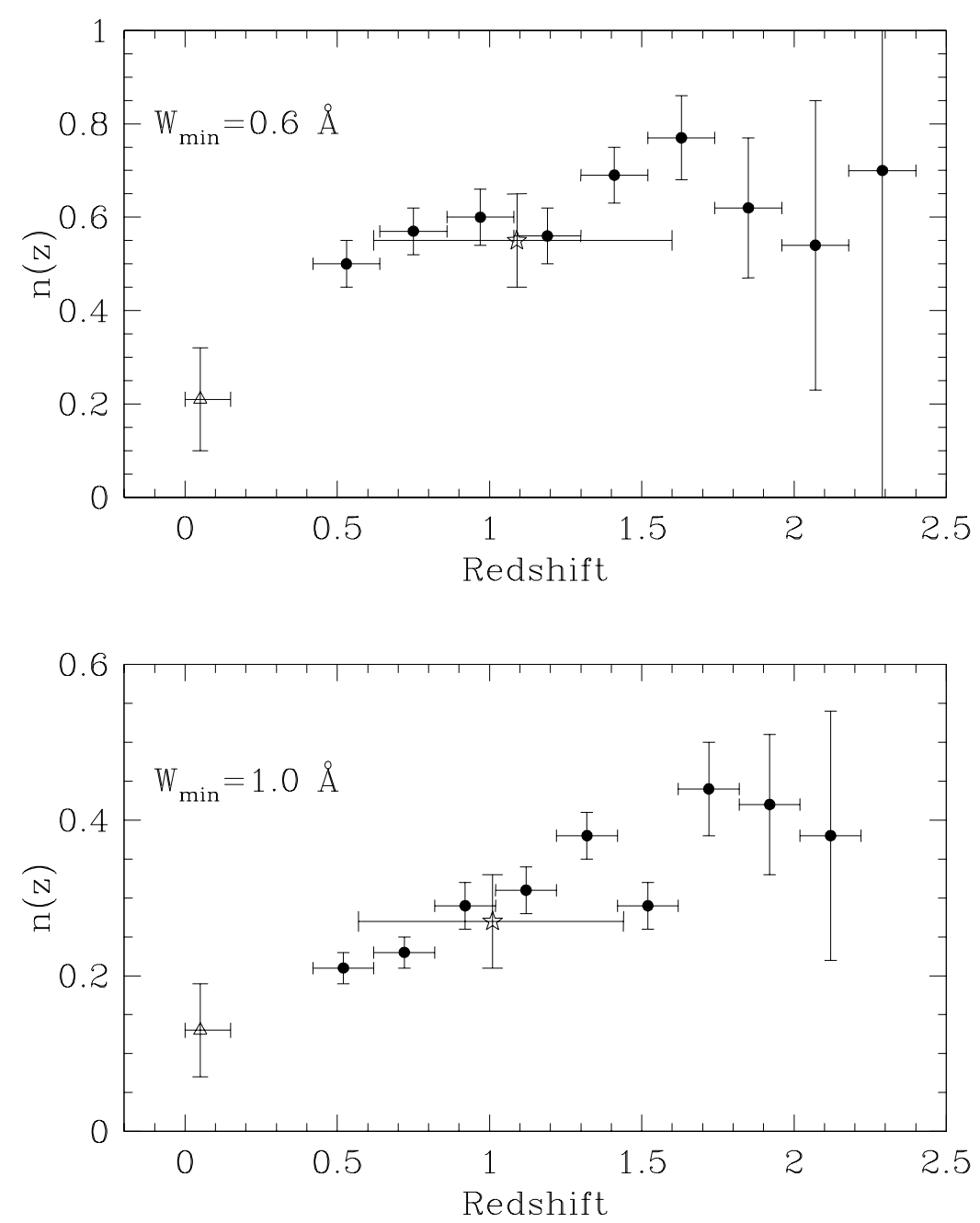

Figure 1. Number density of CORALS MgII absorbers (open stars) for two EW cuts compared with the SDSS-based survey Nestor et al. (2005, filled circles). The low redshift point is taken from Churchill (2001).

In Ellison et al. (2004) we assumed that 50\% of high EW MgII and FeII absorbers would be DLAs (Rao \& Turnshek 2000). At this meeting, Sandhya Rao has reported improved statistics from their low redshift DLA survey and have revised the $50 \%$ confirmation rate of DLAs from strong metal line systems down to 35\%. The implied number density of low redshift DLAs in our MgII sample using this new confirmation rate (and the high redshift $n(z)$ determined by Ellison et al. 2001) is shown in Fig. 2 and is in excellent agreement with the compilation of Storrie-Lombardi \& Wolfe (2000).

\subsection{High redshift DLAs - metallicity}

In the introduction I gave two examples of observational evidence that DLAs may be caused by a dust obscuration bias; both were based on metallicity measurements. The obvious next step is therefore to obtain high resolution spectra of the CORALS DLAs, 


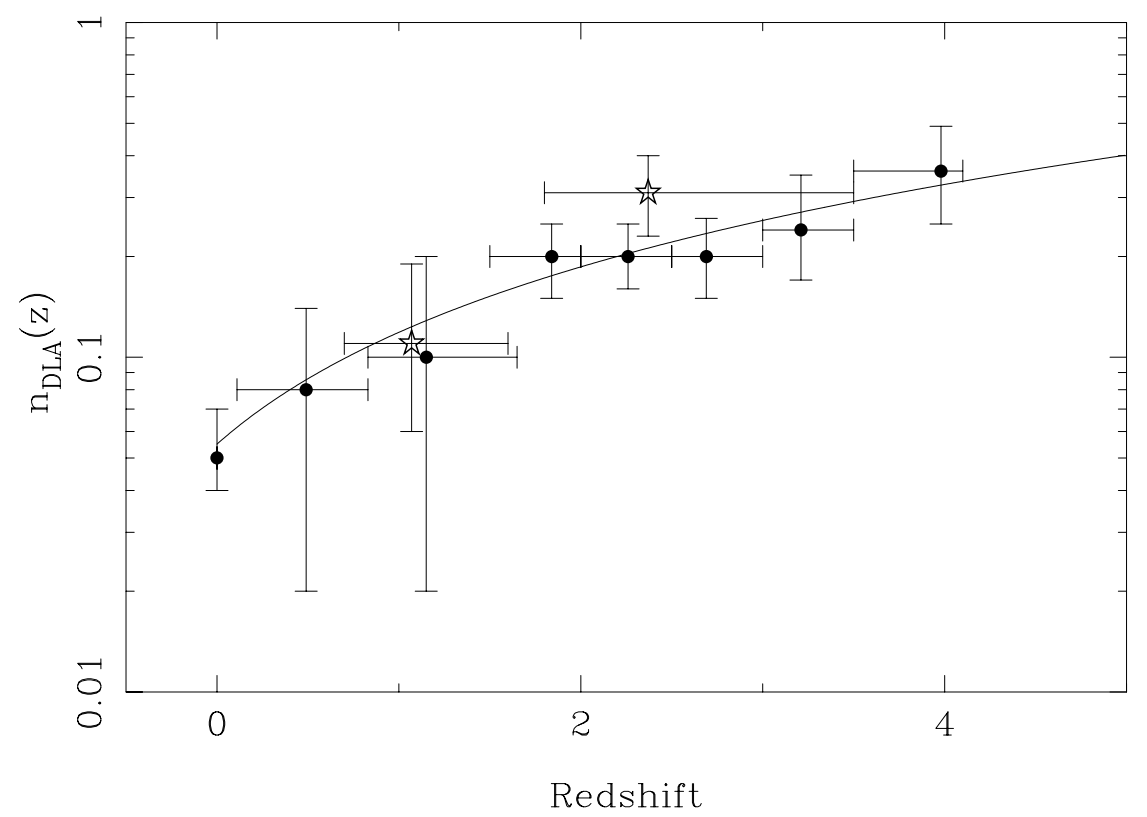

Figure 2. Number density of DLAs from CORALS (open stars): at high redshift from Ellison et al. (2001) and low redshift, as inferred from MgII absorber statistics (Ellison et al. 2004) with the revised DLA confirmation rate of $35 \%$ (Rao, this meeting). Solid points show results of the optical compilation of Storrie-Lombardi \& Wolfe (2000).

in order to determine element abundances. We have recently completed this work (Akerman et al. 2005) using the echelle spectrographs on the VLT (UVES), Keck (ESI), and Magellan (MIKE). We have measured $[\mathrm{Zn} / \mathrm{H}]$ (or obtained upper limits) for 20/22 DLAs in our high- $z$ sample; the last two DLAs are toward a single QSO that is simply too faint even for $8-10-\mathrm{m}$ class telescopes. However, the relatively low $\mathrm{N}(\mathrm{HI})$ in these systems means that it is extremely unlikely that these missed absorbers will change our column density weighted statistics.

In Fig. 3 we compare our Zn abundances (Akerman et al. 2005) with those from the compilation of Kulkarni et al. (2005) and also show the column density weighted mean in the redshift bin $1.8<z<3.5$. The difference between CORALS and the literature values depends somewhat on the treatment of the upper limits, an issue which is discussed more completely by Akerman et al. (2005). In Fig. 3 we show the most conservative case (i.e. the treatment which yields the largest difference between the two samples) where the upper limits are treated as detections. There is a marginally higher metallicity in the CORALS DLAs, but the difference is only 0.2 dex.

\subsection{Estimating extinction from $B-K$ colours}

The results described in the previous sections indicate that the effect of dust in DLA samples is not large; within the error bars most of the absorber statistics are in good agreement with previous surveys. However, as discussed in the introduction, we are confident that some dust is present in DLAs based on $[\mathrm{Zn} / \mathrm{Cr}]$ ratios (and may result in slightly lower number densities in the brightest QSO samples, see $\S 2.1$ ). Based on fitting the spectral slopes of SDSS QSOs, Murphy \& Liske (2004) have recently reported that the reddening associated with DLA galaxies is small: $\mathrm{E}(\mathrm{B}-\mathrm{V})<0.02$. 


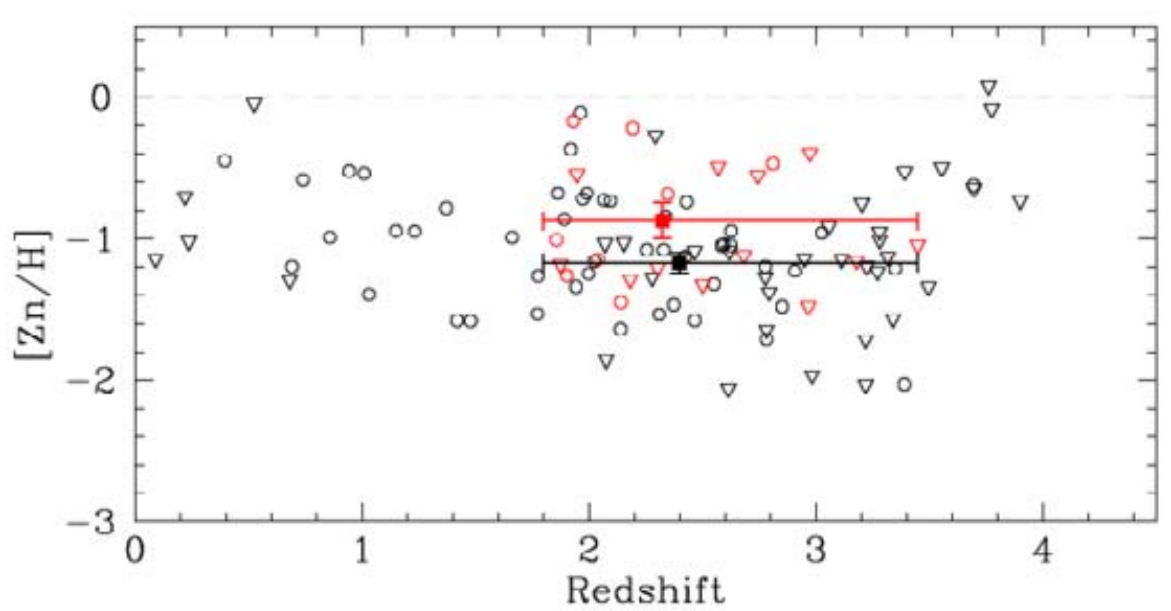

Figure 3. Metallicity of DLAs in the CORALS sample (red symbols, Akerman et al. 2005) and optically selected samples (black symbols from the compilation of Kulkarni et al. 2005). Open circles are detections, downward pointing triangles are upper limits. Binned, column density weighted values (filled points, same colour coding) are shown with bootstrap errors.

In order to determine the reddening in the high $z$ CORALS sample, we have obtained near-simultaneous optical and IR photometry of 46/66 QSOs (including 14 DLAs). The advantage of using optical-IR colours rather than spectral index fitting is that a much wider wavelength baseline can be covered, potentially offering a more sensitive lever on the dust reddening. Although the SDSS sample offers the benefit of a large statistical sample, it is magnitude limited, whereas CORALS is optically complete. The main observational challenge, however, has been to obtain near simultaneous optical and IR photometry for the CORALS QSOs. This is necessary due to potentially rapid variability of radio loud QSOs. The data were taken with the optical (SuSI2) and IR (SofI) imagers on the NTT at La Silla; since these are both mounted at the same focus, we could switch easily and quickly between them during the night.

We have calculated normalised B-K colours which account for effects such as redshift dependence and $B$-band flux suppression due to the DLA Ly $\alpha$ (see Ellison, Hall \& Lira 2005 for more details). A Kolmogorov-Smirnov test on the colours of QSOs with and without DLAs yields only a $25 \%$ probability that the two colour distributions are drawn from the same parent population. In order to quantify the amount of dust implied by this result, we can de-redden the colours of QSOs with DLAs according to an adopted dust recipe. If we assume, for example, that the $\mathrm{E}(\mathrm{B}-\mathrm{V})$ is fixed for every DLA, we find a most probable value of $\mathrm{E}(\mathrm{B}-\mathrm{V})=0.02(0.05)$ for SMC (Milky Way) extinction. If we assume that $\mathrm{E}(\mathrm{B}-\mathrm{V})$ is dependent on $N(\mathrm{H} \mathrm{I})$, we determine a best fit of $\mathrm{E}(\mathrm{B}-\mathrm{V})=$ $N(\mathrm{H} \mathrm{I}) / 2.9 \times 10^{22}$, i.e. a reddening-to-gas ratio some $30-50 \%$ higher than in the SMC.

\section{Bringing it all together}

The presence of dust in DLAs has been well established since the 1980 s when [Zn/Cr] ratios were first shown to be super-Solar. The key question is whether there is sufficient dust to bias our statistics, e.g. reducing the observed number density as well as the inferred gas and metals content. The body of work described above has not provided any 


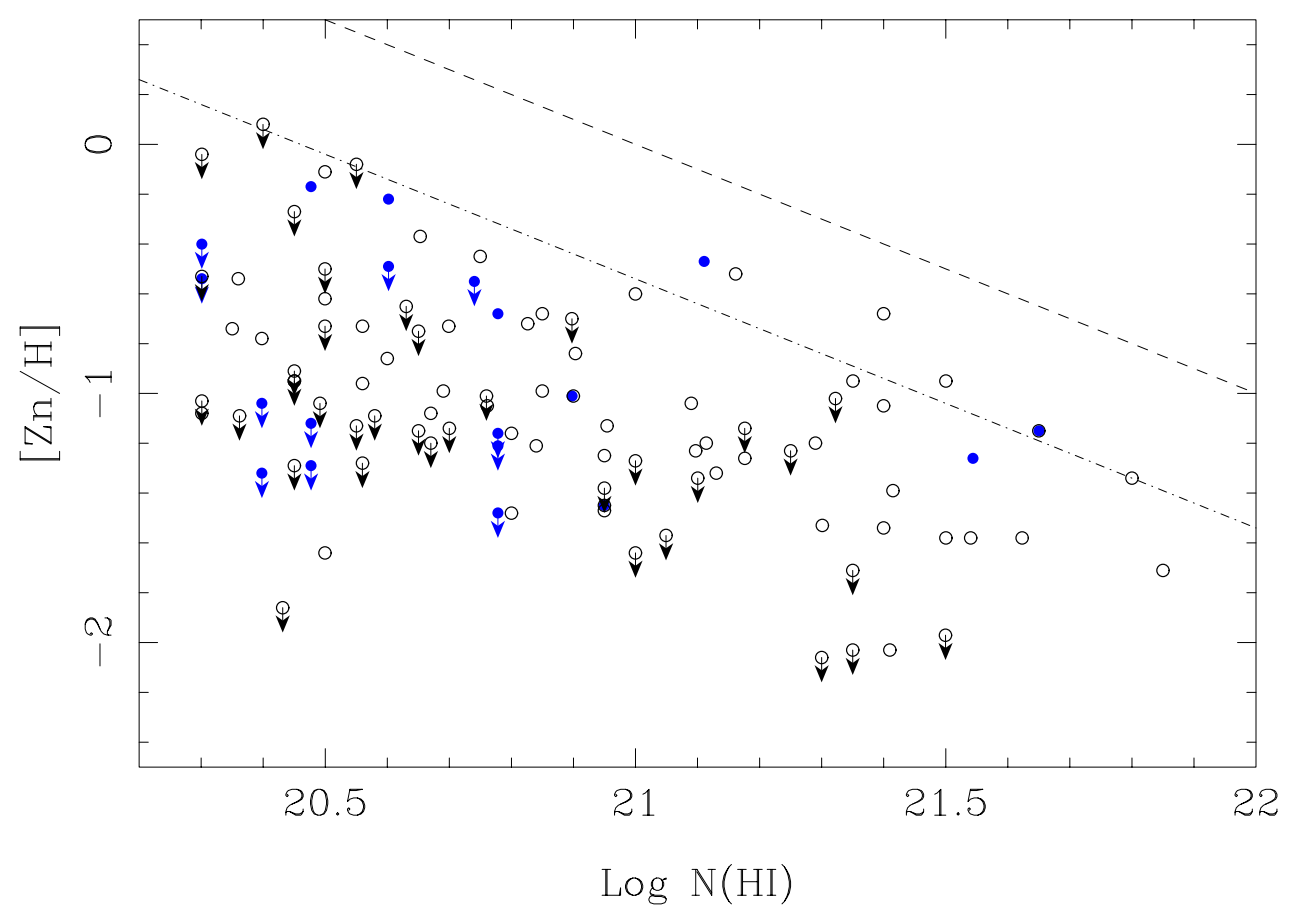

Figure 4. The apparent anti-correlation between metallicity and N(HI) in DLAs identified in optically selected QSO samples (open points) appears to persist in CORALS (filled points). The dashed line corresponds to the dust filter proposed by Prantzos \& Boissier (2000), log N(HI) + $[\mathrm{Zn} / \mathrm{H}]<21$. The dot-dashed line shows the relationship between metallicity and $\mathrm{N}(\mathrm{HI})$ for a Galactic gas-reddening with $\mathrm{E}(\mathrm{B}-\mathrm{V})=0.05$.

grounds for such a concern; all evidence (so far) points to only minor differences between magnitude limited QSO surveys and our optically complete radio-selected one. The lack of dependence of $\Omega_{\mathrm{DLA}}$ and $n(z)$ on magnitude for $B>19.5$ indicates that surveys complete to this limit are unlikely to suffer serious dust selection effects. However, we do see evidence that dust is present, for example the dependence of $n(z)$ and $\Omega_{\mathrm{DLA}}$ on $B$-band magnitude for the brightest QSOs and the slightly redder colours of QSOs with intervening DLAs. For a fixed $\mathrm{E}(\mathrm{B}-\mathrm{V})$ in every DLA and an SMC extinction curve (see Ellison, Hall \& Lira 2005 for other dust 'recipes') we determine $\mathrm{E}(\mathrm{B}-\mathrm{V})<0.05$ at $99 \%$ confidence and a best fit value of 0.02 for SMC extinction. This corresponds to an expected observed frame $A_{V} \sim 0.25$ magnitudes, a relatively small effect. Although the number of DLAs in the high $z$ CORALS sample is modest $(\sim 20)$ a second survey by the UCSD group has now almost doubled the number of radio-selected QSOs (Jorgenson et al. in preparation) Encouragingly, the combined samples, now with a redshift path of $\Delta z \sim 100$, yield a value of $\Omega_{\mathrm{DLA}}$ in good agreement with CORALS and optically selected samples.

The apparent anti-correlation between $[\mathrm{Zn} / \mathrm{H}]$ and $N(\mathrm{H} \mathrm{I})$ is often attributed to dust bias, but in Fig. 4 we show that none of the CORALS DLAs (which, we recall, are not subject to such selection effects) appear in the 'dust-forbidden' region. However, high $N(\mathrm{H} \mathrm{I})$, high $[\mathrm{Zn} / \mathrm{H}]$ values are expected to be quite rare since these are the sight-lines which presumably pass through galaxies at the smallest impact parameters. The simulations of Churches et al. (2004), presented by Alistair Nelson at this meeting, indicate that $\lesssim 9 \%$ (depending upon spin parameter) of DLAs will be in this region, so it maybe 


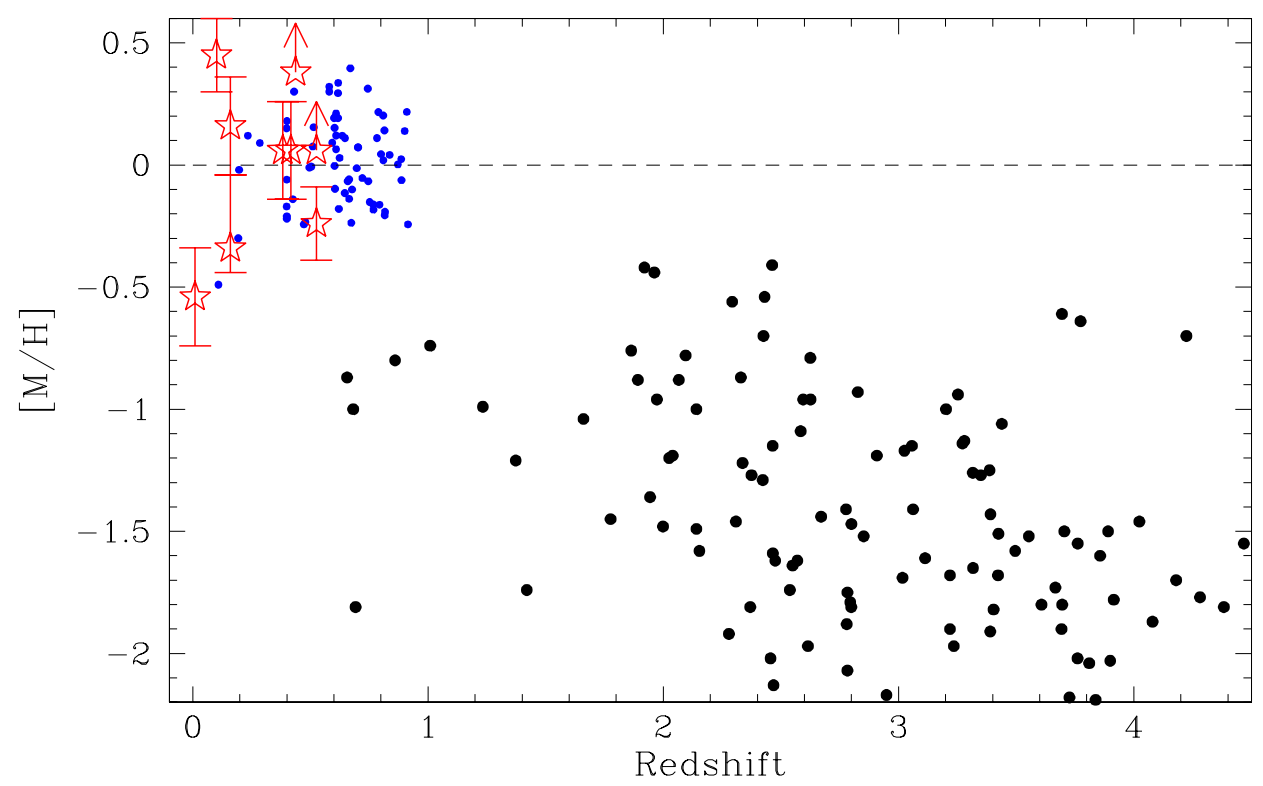

Figure 5. The metallicity of DLAs (black/large points at $z>0.5$ ), emission line galaxies (blue/small points at $z<1$ ) and absorption selected galaxies (DLAs and sub-DLAs) measured in emission (open stars). DLA galaxies have $\sim$ Solar metallicities when measured in emission, compared with uniformly sub-Solar abundances when measured in absorption.

premature to interpret the lack of high $N(\mathrm{H} \mathrm{I})$, high metallicity CORALS DLAs as evidence that the anti-correlation is not caused by dust. However, it is interesting to calculate what $\mathrm{E}(\mathrm{B}-\mathrm{V})$ is implied by the envelope of DLA detections. If we assume a Galactic reddening to gas ratio which scales as metallicity, i.e. $\mathrm{E}(\mathrm{B}-\mathrm{V})=N(\mathrm{H} \mathrm{I})$ $\times 10^{[\mathrm{Zn} / \mathrm{H}]} / 5.9 \times 10^{21}$, the empirical cut-off suggested by Prantzos \& Boissier (2000) corresponds to quite a large reddening: $\mathrm{E}(\mathrm{B}-\mathrm{V})=0.17$. On the other hand, our reddening results, for a Milky Way extinction curve, give $\mathrm{E}(\mathrm{B}-\mathrm{V})=0.05$. Drawing the corresponding relation between $N(\mathrm{H} \mathrm{I})$ and $[\mathrm{Zn} / \mathrm{H}]$ in Fig. 4 yields a line which fits snugly along the detections. This indicates that if the anti-correlation in Fig. 4 is caused by dust then the reddening may be small.

The CORALS results have not provided any indication that the typically low metallicities in DLAs are due to dust bias. Although the low redshift CORALS sample does not have measured metallicities, it is interesting to speculate what alternative explanations there may be for the lack of strong evolution in $[\mathrm{Zn} / \mathrm{H}]$ down to $z \sim 0.5$. Two contributions at this meeting, I believe, are important clues to this outstanding question. First, Marten Zwaan has used $21 \mathrm{~cm}$ maps of galaxies in the local Universe, combined with assumptions of their metallicity and abundance gradients, to infer the $z=0$ mean weighted metallicity. The value found is $\sim 1 / 3$ Solar, indicating that we should perhaps not expect the DLA metallicities to evolve to Solar at low $z$. Second, Hsiao-Wen Chen has presented her results on the emission line metallicities of galaxies associated with DLA absorbers at low $z$. Chen's results, combined with similar work from Schulte-Ladbeck et al. (2004) and Ellison, Kewley \& Mallen-Ornelas (2005), are shown in Fig. 5. We can see that when measured from emission lines, DLAs have typically Solar abundances, similar to other bright emission line galaxies at $z<1$. These results indicate that sub-Solar abundances 
in DLAs may be due, at least in part, to substantial galaxy-QSO impact parameters. Whereas Solar metallicities in emission line galaxy samples are weighted by luminosity, DLA measurements are weighted by HI cross-sectional area. The tenet that DLAs are a representative probe of gas and metals is then secure without the need to invoke grave observational biases. Nonetheless, larger complete surveys are desirable to improve the statistics derived from CORALS and I think that optically faint X-ray selected QSOs (which are being found in large number by surveys with XMM and Chandra) provide the most promising prospect in this direction.

\section{Acknowledgements}

I am extremely grateful to my past and present collaborators who have contributed to various aspects of the CORALS survey: Max Pettini, Chris Akerman, Chris Churchill, Pat Hall, Isobel Hook, Carole Jackson, Paulina Lira, Samantha Rix, Peter Shaver, Chuck Steidel, Jasper Wall, and Lin Yan. I am fortunate to have such insightful and productive colleagues with whom it is a joy to work. I would also like to thank the organisers of this meeting which has combined a fascinating destination with a stimulating programme.

\section{References}

Akerman, C. J., Ellison, S. L., Pettini, M., Steidel, C. C., 2005, A\&A, submitted Boissé, P., Le Brun, V., Bergeron, J., Deharveng, J.-M., 1998, A\&A, 333, 841

Churches, D. K., Nelson, A. H., \& Edmunds, M. G., 2004 MNRAS, 347, 1234

Churchill, C. W., 2001, ApJ, 560, 92

Ellison, S. L., Churchill, C. W., Rix, S. A., Pettini, M., 2004, ApJ, 615, 118

Ellison, S. L., Hall, P., Lira, P., 2005, AJ, submitted

Ellison, S. L., Kewley, L. J., \& Mallén-Ornelas, G., 2005, MNRAS, 357, 354

Ellison, S. L., Yan, L., Hook, I., Pettini, M., Wall, J., Shaver, P., 2001, A\&A, 379, 393

Fall, S. M., Pei, Y., 1993, ApJ, 402, 479

Jackson, C. A., Wall, J. V., Shaver, P. A., Kellermann, K. I., Hook, I. M., Hawkins, M. R. S., 2002, A\&A, 386, 97

Kulkarni, V., Fall, S. M., Lauroesch, J. T., York, D. G., Welty, D. E., Khare, P., Truran, J. W., 2005, ApJ, 618, 68

Murphy, M. T., Liske, J., 2004, MNRAS, 345, L31

Nestor, D. B., Turnshek, D. A., Rao, S. M., 2005, ApJ, in press

Ostriker, J. P., Heisler, J., 1984, ApJ, 278, 1

Prantzos, N., Boissier, S., 2000, MNRAS, 315, 82

Rao, S. M., Turnshek, D. A., 2000, ApJS, 130, 1

Schulte-Ladbeck, R. E., Rao, S. M., Drozdovsky, I. O., Turnshek, D. O., Nestor, D. B., Pettini, M., 2004, ApJ, 600, 613

Storrie-Lombardi, L., Wolfe, A. M., 2000, ApJ 543, 552

Vladilo, G., Peroux, C., 2005, A\&A, submitted 